\title{
Instruments to identify psychosocial risk factors at work: a systematic review
}

\section{Instrumentos para identificar los factores de riesgos psicosociales en el trabajo: una revisión sistemática}

\author{
SUÁREZ-REYES, Susana†*, AGUILAR-MORALES, Norma and MAGAÑA-MEDINA, Deneb Elí \\ Universidad Juárez Autónoma de Tabasco, Academic Division of Administrative Economic Sciences, Mexico.
}

ID 1 ${ }^{\text {st }}$ Author: Susana, Suárez-Reyes / ORC ID: 0000-0003-0153-1068, CVU CONACYT ID: 838292

ID $1^{\text {st }}$ Co-author: Norma, Aguilar-Morales / ORC ID: 0000-0002-7292-3029, CVU CONACYT ID: 478554

ID $2^{\text {nd }}$ Co-author: Deneb Elí, Magaña-Medina / ORC ID: 0000-0002-8579-596X, CVU CONACYT ID: 253765

DOI: $10.35429 / J L D E .2020 .7 .4 .27 .39$

Received July 20, 2020; Accepted December 30, 2020

\begin{abstract}
The human factor within organizations is exposed to different types of risks, especially those that cause damage to their physical and psychological health, which if not identified and treated in time can generate irreversible damage to them. In recent years, investigations have been carried out that address the subject, in which different instruments have been implemented to identify and evaluate this phenomenon, so the purpose of this systematic review article was to analyze the instruments that allow identifying the factors of Psychosocial risks at work (FPR) in Latin America. The information search included databases on the web, such as Redalyc, Dialnet, Scielo and ProQuest. Three people independently selected the articles for review, in a period from 2015 to 2020, where the methodological quality of each of them was evaluated, as well as the inclusion and exclusion criteria considered for the research. It was observed that in recent years different methods and instruments have been implemented to evaluate this phenomenon from different areas, so it is important to determine which are the optimal instruments to identify FPR in workers.
\end{abstract}

Psychosocial risk, Scale of psychosocial risk, Latin America

\begin{abstract}
Resumen
El factor humano dentro de las organizaciones se encuentra expuesto a diferentes tipos de riesgos, sobre todo a los que generan daños en su salud física y psicológica, los cuales si no se identifican y tratan a tiempo pueden generar daños irreversibles en ellos. En los últimos años, se han realizado investigaciones que abordan el tema, en las que se han implementado diferentes instrumentos para identificar y evaluar dicho fenómeno, por lo que el propósito de este artículo de revisión sistemática fue analizar los instrumentos que permiten identificar los factores de riesgos psicosociales laborales (FRP) en América Latina. La búsqueda de información incluyó bases de datos en la web, tal como Redalyc, Dialnet, Scielo y ProQuest. Tres personas seleccionaron los artículos para su revisión de manera independiente, en un periodo de 2015 a 2020, donde se evaluaron la calidad metodológica de cada uno de ellos, así como los criterios de inclusión y exclusión considerados para la investigación. Se pudo observar que en los últimos años se han implementado diferentes métodos e instrumentos para evaluar este fenómeno desde diferentes ámbitos, por lo que resulta importante determinar cuáles son los instrumentos óptimos para identificar los FRP en los trabajadores.
\end{abstract}

Riesgo psicosocial, Escalas de riesgo psicosocial, América Latina

Citation: SUÁREZ-REYES, Susana, AGUILAR-MORALES, Norma and MAGAÑA-MEDINA, Deneb Elí. Instruments to identify psychosocial risk factors at work: a systematic review. Journal- Labor and Demographic economic. 2020. 4-7:27-39.

\footnotetext{
* Correspondence to Author (Email: susy.93suarez@gmail.com)

$\dagger$ Researcher contributing first author.
} 


\section{Introduction}

In recent years, human resource management has become increasingly important, since it is people who generate competitive advantages for organizations.

However, despite the fact that the human factor has been considered a valuable resource for organizations and that some of the traditional risks have been reduced with attention to the safety of workers, other risks continue to affect their health, as it has generated an increase in new occupational diseases without taking adequate prevention, protection and control measures (International Labor Organization [ILO], 2013b).

Human resources are frequently exposed to various factors in their workplace and in their social environment, which have generated problems in their physical, mental and emotional health, such as excessive workloads, role ambiguity, lack of social support, work-family conflict, among others, which are known as psychosocial risk factors that affect the productivity of the organization (European Agency for Safety and Health at Work [EUOSHA], 2020).

This requires that studies be carried out to identify and evaluate the psychosocial risk factors caused by the different changes that organizations face, in order to prevent such risks and promote healthy environments for workers, considering work interactions, conditions organizational and performance environment, in order to guarantee productivity and organizational growth (Muñoz, Orellano \& Hernández, 2018).

This article includes aspects related to occupational psychosocial risk factors and the importance that attention and assessment of these risks have generated in Latin American countries, so the objective of this research was to carry out a systematic review to determine and analyze the instruments that make it possible to identify and assess psychosocial risk factors and take the necessary measures with respect to them.

\section{Psychosocial risk factors}

Psychosocial factors at work are not a new phenomenon, since they have always been present in organizations, but their importance had been put in the background due to the presence of other occupational hazards that are more visible in workers.

Their study has evolved since the crisis of the 1970s, when a new capitalist accumulation regime was established, and they have been officially considered since 1984 by the ILO (Moreno, 2011; Neffa, 2015).

However, due to the relevance that the subject of occupational health has acquired in recent years, the study of psychosocial risk factors has been increasing, since it is necessary to analyze the interactions that the individual has with the work environment, where they interact genetic, psychological, social components and circumstances that can affect the health and wellbeing of workers, since no person who works will be exempt from being affected by them (Rodríguez, 2009).

Currently, people are exposed to various damages generated by their jobs, such as stress, burnout, workplace violence, fatigue, sleep disorders, addictions, alcoholism, among others, so the psychosocial risks at work began to be object of concern in various parts of the world, beyond traditional approaches to occupational safety and health (Henry, 2017).

Psychosocial risk factors are those characteristics of working conditions that affect people's health, through psychological and physiological mechanisms called stress, such as their internal and external environment, work activities and activities. organization conditions, which interact with the worker's capacities, needs, culture and personal situation outside of work, (Joint ILO / WHO Committee, 1984; National Institute for Occupational Safety and Health [INSST], nd; ILO, 2013a).

Sometimes, psychosocial risk factors are convenient for the development of work activities and for the quality of work life and personal development of workers, but in most cases, these factors are unfavorable and have harmful consequences for their health and for their physical and mental well-being (Ruiz, Pulla, Parra, Parra and Zamora, 2017). 
Among the effects generated by these factors, we can mention work overload, interpersonal conflicts, low levels of participation in company decisions, role ambiguity, job insecurity, as well as different mental and health disorders, such as alterations in the sleep, anxiety, depression, work accidents, absenteeism and occupational diseases (GilMonte, López-Vílchez, Llorca-Rubio and Sánchez-Piernas, 2016).

However, not all workers react in the same way to the psychosocial risk factors to which they are exposed, and this will depend on the perceptions and experiences of each of them (Gil-Monte, 2012), as well as their own skills to handle the work situations they perform and the environment that surrounds them (Rodríguez, 2009).

It is inevitable that workers are exempt from various occupational risks, so it is necessary for organizations to seek and guarantee their protection and safety, in order to avoid damage to the health of personnel, as well as damage to the organization.

Therefore, it is necessary to identify and evaluate the psychosocial risk factors that negatively influence productivity and well-being at work, in order to design and implement strategies to counteract their negative effects, as well as strategies that allow satisfying the organizational and personal needs and objectives of workers (Muñoz, Orellano and Hernández, 2018).

\section{Identification and evaluation of psychosocial risk factors in Latin America}

Different international organizations such as the ILO, the World Health Organization (WHO), the EU-OSHA, the Organization for Economic Cooperation and Development (OECD), among others, have been concerned about the psychosocial aspects generated by work in people, classifying them as one of the world priorities to be treated, since psychosocial factors and stress generate negative effects on health, such as musculoskeletal disorders, metabolic dysfunctions, cardiovascular, physical and mental problems (Leka and Jain, 2010).
However, in Latin American countries, issues related to occupational safety and health have received little attention, since developed countries have an advantage of between 20 and 40 years over the study and attention to factors of psychosocial risks, while in Latin America in the last 10 years there have been advances in legal issues, such as rules, laws and regulations, as well as some academic research that has implemented standardized questionnaires, which are developed or adapted in countries such as Colombia, Peru, Chile, Mexico and Argentina (Juárez-García, 2015).

Such is the case of Mexico, that until October 23, 2018, the need to identify, evaluate and control psychosocial risk factors was regulated, through the Official Mexican Standard NOM-035-STPS-2018, which includes strategies for the identification, analysis and prevention of psychosocial risk factors, workplace violence, and the promotion of a favorable organizational environment (Ministry of Labor and Social Security [STPS], 2019).

In Colombia, psychosocial risk factors are regulated by legal statutes and the Ministry of Social Protection of Colombia, so the evaluation of these factors is based on Resolution 2646 of 2008, which was issued in the same year, as well as the psychosocial risk battery, which is an instrument designed by the Pontificia Universidad Javeriana and the Ministry of Social Protection of Colombia (Salamanca, Pérez, Infante and Olarte, 2019), with which it is possible to identify, evaluate, prevent, intervene and monitor permanently exposing people to psychosocial risk factors at work, as well as determining the origin of pathologies caused by occupational stress (Ministerio de la Protección Social, 2008).

In Argentina the situation is different, since the psychosocial risks generated at work have not been taken into due importance by the corresponding authorities, since these aspects are considered minor damages and are not mentioned or recognized as such in the legislation on the matter. risks at work, but is limited to risks that damage the physical health of workers, without considering the psychic and mental dimensions (Neffa, 2015, 2017). 
These are just some countries in Latin America that have deficiencies in the prevention and assessment of psychosocial risk factors, which until a few years ago began to raise awareness of their importance to ensure the wellbeing of workers, therefore The regulatory policies of the countries should be the basis for organizations to manage these factors, through the different instruments that serve to diagnose and evaluate them (Potter, O'Keeffe, Leka, Webber, and Dollard, 2019).

\section{Methodology}

The methodology of this research is based on a review of the scientific literature, in relation to the instruments to identify the factors of occupational psychosocial risks, taking into account the inclusion criteria, such as articles in Spanish published in scientific journals in a period of 2015 to 2020, which include instruments to identify psychosocial risk factors at work, with samples from Latin American countries.

The search and review chain procedure was carried out by three people independently, in the period from 2015 to 2020, in databases such as Redalyc, Dialnet, Scielo and ProQuest, which are databases with scientific articles in Spanish, with publications from Latin America.

To carry out the initial search of the articles, terms such as "psychosocial risk", "psychosocial risk scales" and "Latin America" were used, which were entered into the databases directly or in combination, in order to find publications relevant to the content of this review, within the established search period (Figure 1).

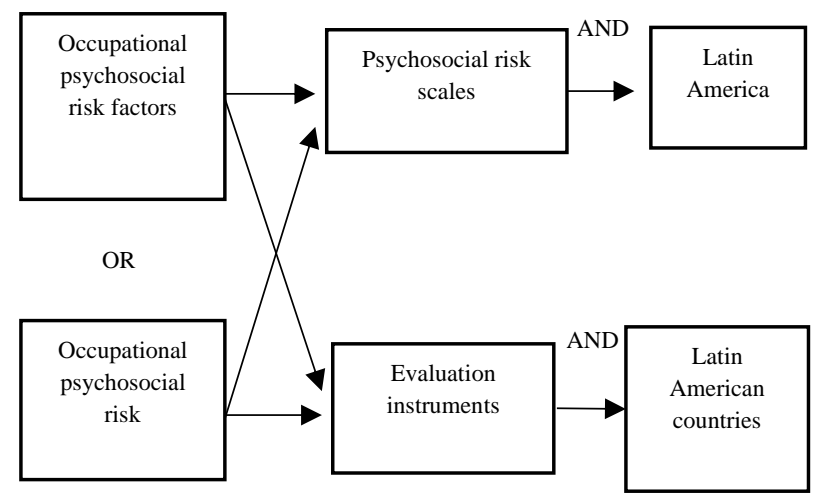

Figure 1 Keywords and Boolean operators used to search for information

\section{Selection of documents}

The articles were selected through a first filter, in which the following inclusion criteria were considered: a) articles published in scientific journals in a period from 2015 to 2020, b) publications of studies carried out with samples from Latin American countries , c) articles published in Spanish, d) articles presenting the research instrument implemented, e) articles that used instruments to identify psychosocial risk factors at work and f) articles with a quantitative research approach.

The following exclusion criteria were established: a) theses, books, abstracts of congresses and presentations, b) publications from countries other than Latin America, c) theoretical articles, d) duplicate publications, and e) articles with a research focus qualitative and mixed.

As a second filter, a manual review of the selected articles was performed, to verify that they met the specified criteria and that they were relevant to the research, which was carried out through meetings between the reviewers.

In this search, all those articles that were repeated in the selected databases, theoretical articles and articles that did not have a quantitative research approach, as well as those articles that did not report the psychosocial risk scales analyzed were excluded (Figure 2).

Finally, information was obtained from each of the articles, such as the title, authors, year of publication, country, study design, as well as the scales and main psychosocial risk factors.

With this information, we proceeded to analyze the instruments implemented to assess psychosocial risk factors in Latin American countries. 
December 2020 Vol.4 No.7 27-39

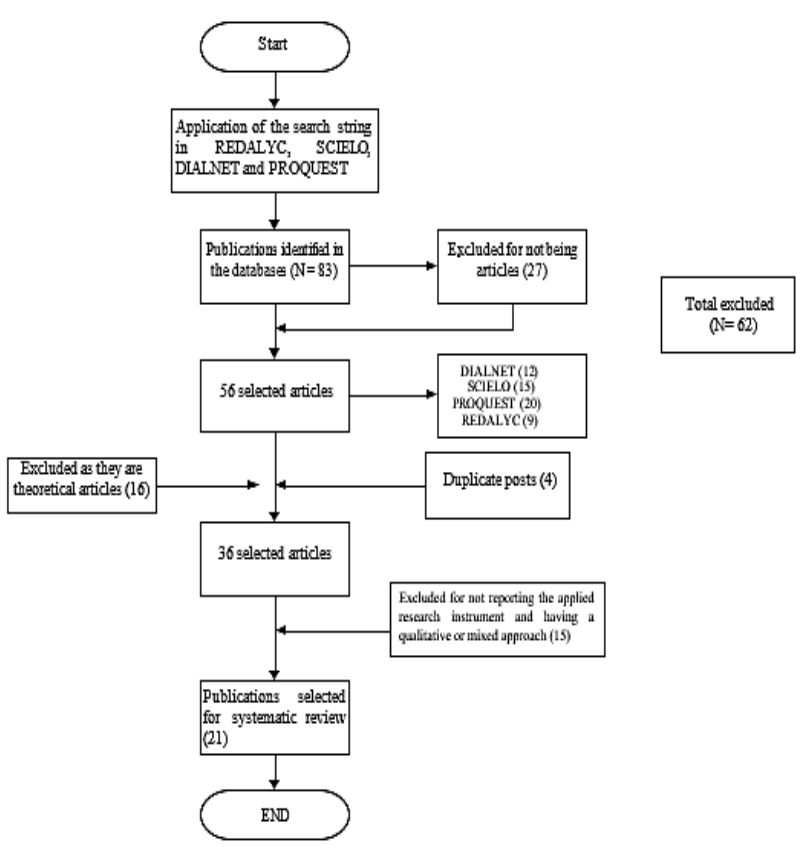

Figure 2 Selection process of the articles included in the systematic review

\section{Results}

The articles selected for the present systematic review responded to the indicated inclusion criteria, resulting in a total of 21 articles (Table $1)$.

The prevailing psychosocial factors were double presence (33.3\%), followed by psychosocial demands (28.6\%), work conditions (23.8\%), and workload (19\%).

\begin{tabular}{|c|c|c|c|}
\hline $\begin{array}{l}\text { Code/ } \\
\text { Base }\end{array}$ & Title & $\begin{array}{l}\text { Author/ } \\
\text { Country }\end{array}$ & $\begin{array}{l}\text { Main FRP } \\
\text { found }\end{array}$ \\
\hline $\begin{array}{l}\text { A1 } \\
\text { DIALNET }\end{array}$ & $\begin{array}{l}\text { Psychosocial factors } \\
\text { and symptoms of } \\
\text { occupational stress in } \\
\text { workers in the } \\
\text { production area of a } \\
\text { metal-mechanic } \\
\text { company in El Salto, } \\
\text { Jalisco }\end{array}$ & $\begin{array}{l}\text { Aranda, Mares, } \\
\text { Ramírez and } \\
\text { Rojas (2016). } \\
\text { Mexico }\end{array}$ & $\begin{array}{l}\text { Labor } \\
\text { requirements } \\
(90.7 \%), \\
\text { Workload } \\
(86.7 \%), \quad \text { Job } \\
\text { role and career } \\
\text { development } \\
(77.3 \%), \\
\text { Workplace } \\
\text { conditions } \\
(70.7 \%) \quad \text { and } \\
\text { task } \\
\text { characteristics } \\
(66.7 \%) .\end{array}$ \\
\hline $\begin{array}{l}\text { A2 } \\
\text { DIALNET }\end{array}$ & $\begin{array}{l}\text { Diagnosis of } \\
\text { psychosocial risk } \\
\text { factors in the } \\
\text { workplace in the } \\
\text { company Miramar in } \\
\text { the municipality of } \\
\text { Maicao in the Guajira }\end{array}$ & $\begin{array}{lr}\text { Muñoz, } & \text { Pitre } \\
\text { and } & \text { Amaya } \\
(2017) . & \\
\text { Colombia } & \end{array}$ & $\begin{array}{l}\text { Role Clarity } \\
(25.67 \%) \text {, } \\
\text { Training } \\
(41.67 \%) \text { and } \\
\text { Participation } \\
\text { and Change } \\
\text { Management } \\
(16.67 \%) \text { are at } \\
\text { high risk. }\end{array}$ \\
\hline $\begin{array}{l}\text { A3 } \\
\text { PROQUEST }\end{array}$ & $\begin{array}{l}\text { Identification } \\
\text { psychosocial risk } \\
\text { factors in a high } \\
\text { complexity clinic }\end{array}$ & $\begin{array}{l}\text { Bobadilla, } \\
\text { Garzón and } \\
\text { Charria (2018). } \\
\text { Colombia }\end{array}$ & $\begin{array}{l}\text { Work demands } \\
\text { and control over } \\
\text { work; at the } \\
\text { extralaboral } \\
\text { level: Time } \\
\text { away from } \\
\text { work, economic } \\
\text { situation of the } \\
\text { family group, } \\
\text { characteristics } \\
\text { of the home and } \\
\text { its environment, } \\
\text { and home-work } \\
\text { displacement. }\end{array}$ \\
\hline
\end{tabular}

ISSN-On line: 2524-2067

RINOE $^{\circledR}$ All rights reserved.

\begin{tabular}{|c|c|c|c|}
\hline $\begin{array}{l}\text { Code/ } \\
\text { Base }\end{array}$ & Title & $\begin{array}{l}\text { Author/ } \\
\text { Country }\end{array}$ & $\begin{array}{l}\text { Main FRP } \\
\text { found }\end{array}$ \\
\hline $\begin{array}{l}\text { A4 } \\
\text { REDALYC }\end{array}$ & $\begin{array}{l}\text { Psychosocial factors } \\
\text { at work and their } \\
\text { relationship to the } \\
\text { dual presence of } \\
\text { women workers in } \\
\text { primary health care }\end{array}$ & $\begin{array}{l}\text { Toia, Mattos, } \\
\text { Figueroa, } \\
\text { Aguilar, Chavez } \\
\text {, Del Carpio, } \\
\text { Gamarra, } \\
\text { García, } \\
\text { Miranda, } \\
\text { Romero and } \\
\text { Muñoz (2018). } \\
\text { Peru }\end{array}$ & $\begin{array}{l}\text { Double } \\
\text { presence } \\
(93.9 \%), \\
\text { Psychological } \\
\text { requirements } \\
(59.2 \%), \text { Active } \\
\text { work (93.2\%), } \\
\text { Social support } \\
(100 \%)\end{array}$ \\
\hline $\begin{array}{l}\text { A5 } \\
\text { REDALYC }\end{array}$ & $\begin{array}{l}\text { Psychological well- } \\
\text { being, stress and } \\
\text { psychosocial factors } \\
\text { in workers of } \\
\text { government } \\
\text { institutions in Jalisco, } \\
\text { Mexico }\end{array}$ & $\begin{array}{lr}\text { Saldaña, } & \text { Polo, } \\
\text { Gutiérrez } & \text { and } \\
\text { Madrigal } & \\
(2020) . & \\
\text { Mexico } & \end{array}$ & $\begin{array}{l}\text { Control, } \\
\text { autonomy and } \\
\text { workload, } \\
\text { conditions in the } \\
\text { work } \\
\text { environment } \\
\text { and limited } \\
\text { training. } \\
\text { The FRPS were } \\
\text { related to Stress } \\
\text { and Burnout. }\end{array}$ \\
\hline $\begin{array}{l}\text { A6 } \\
\text { SCIELO }\end{array}$ & $\begin{array}{l}\text { Psychosocial risk } \\
\text { factors and perceived } \\
\text { stress in university } \\
\text { teachers }\end{array}$ & $\begin{array}{l}\text { Tacca and } \\
\text { Tacca (2019). } \\
\text { Peru }\end{array}$ & $\begin{array}{l}\text { Stress is } \\
\text { positively } \\
\text { related to } \\
\text { psychological } \\
\text { demands, active } \\
\text { work, double } \\
\text { presence and } \\
\text { compensation. }\end{array}$ \\
\hline $\begin{array}{l}\text { A7 } \\
\text { SCIELO }\end{array}$ & $\begin{array}{lr}\text { Psychosocial risk } \\
\text { factors and job } \\
\text { satisfaction } & \text { in } \\
\text { seasonal workers in } \\
\text { Chile }\end{array}$ & $\begin{array}{l}\text { Palomo, } \\
\text { Carrasco, } \\
\text { Bastías, Méndez } \\
\text { and Jiménez } \\
(2015) \text {. } \\
\text { Chile }\end{array}$ & $\begin{array}{l}\text { The factors } \\
\text { Dual presence, } \\
\text { and Active work } \\
\text { and } \\
\text { development } \\
\text { possibilities had } \\
\text { a high risk level. } \\
\text { FRPs are } \\
\text { negatively } \\
\text { associated with } \\
\text { job satisfaction. }\end{array}$ \\
\hline $\begin{array}{l}\text { A8 } \\
\text { SCIELO }\end{array}$ & $\begin{array}{l}\text { Diagnosis of the } \\
\text { perceptions } \\
\text { psychosocial risk } \\
\text { factors in the work of } \\
\text { personnel in a } \\
\text { manufacturing } \\
\text { industry }\end{array}$ & $\begin{array}{l}\text { Luna-Chávez, } \\
\text { Anaya-Velasco } \\
\text { and Ramírez- } \\
\text { Lira (2019). } \\
\text { Mexico }\end{array}$ & $\begin{array}{l}\text { Work Content } \\
(55.2 \%) \text { and } \\
\text { Work Context } \\
(40.2 \%) \text {, } \\
\text { making them } \\
\text { likely } \\
\text { psychosocial } \\
\text { risk factors for } \\
\text { Psychic } \\
\text { Attrition. }\end{array}$ \\
\hline $\begin{array}{l}\text { A9 } \\
\text { PROQUEST }\end{array}$ & $\begin{array}{l}\text { Psychosocial risk in } \\
\text { the nursing staff } \\
\text { Emergency service at } \\
\text { the University } \\
\text { Hospital } \\
\text { Cartagena }\end{array}$ & $\begin{array}{l}\text { Bustillo- } \\
\text { Guzmán, Rojas- } \\
\text { Meriño, } \\
\text { Sánchez- } \\
\text { Camacho, } \\
\text { Sánchez-Puello, } \\
\text { Montalvo- } \\
\text { Prieto and } \\
\text { Rojas-López } \\
\text { (2015). } \\
\text { Colombia }\end{array}$ & $\begin{array}{l}\text { High levels of } \\
\text { risk in } \\
\text { Personnel } \\
\text { Management } \\
(98 \%) \text { Task } \\
\text { Content }(75 \%) \\
\text { and } \\
\text { Organisation of } \\
\text { Working Time } \\
(56.3 \%)\end{array}$ \\
\hline $\begin{array}{l}\text { A10 } \\
\text { REDALYC }\end{array}$ & $\begin{array}{l}\text { Psychosocial factors, } \\
\text { psychological stress } \\
\text { and burnout in } \\
\text { nursing: a model of } \\
\text { trajectories }\end{array}$ & $\begin{array}{l}\text { Brito-Ortíz, } \\
\text { Juárez-García, } \\
\text { Nava-Gómez, } \\
\text { Castillo-Pérez } \\
\text { and Brito-Nava } \\
\text { (2019) } \\
\text { Mexico }\end{array}$ & $\begin{array}{l}\text { We found high } \\
\text { levels of social } \\
\text { support, labor } \\
\text { control, and } \\
\text { enthusiasm for } \\
\text { work; moderate } \\
\text { degrees of } \\
\text { psychological } \\
\text { demand and } \\
\text { psychological } \\
\text { stress; and low } \\
\text { levels of } \\
\text { psychological } \\
\text { burnout, } \\
\text { indolence, and } \\
\text { guilt. }\end{array}$ \\
\hline
\end{tabular}

SUÁREZ-REYES, Susana, AGUILAR-MORALES, Norma and MAGAÑA-MEDINA, Deneb Elí. Instruments to identify psychosocial risk factors at work: a systematic review. Journal- Labor and Demographic economic. 2020 


\begin{tabular}{|c|c|c|c|}
\hline $\begin{array}{l}\text { Code/ } \\
\text { Base }\end{array}$ & Title & $\begin{array}{l}\text { Author/ } \\
\text { Country }\end{array}$ & $\begin{array}{l}\text { Main FRP } \\
\text { found }\end{array}$ \\
\hline $\begin{array}{l}\text { A11 } \\
\text { PROQUEST }\end{array}$ & $\begin{array}{l}\text { Psychosocial factors } \\
\text { and r Burnout } \\
\text { syndrome in general } \\
\text { practitioners } \quad \text { in } \\
\text { Tepic, r Nayarit } \\
\text { (Mexico) }\end{array}$ & $\begin{array}{l}\text { Aranda, } \\
\text { Barraza, } \\
\text { Romero, } \\
\text { Quinonez, } \\
\text { Ceniceros, } \\
\text { González and } \\
\text { Esparza (2015). } \\
\text { Mexico }\end{array}$ & $\begin{array}{l}\text { Essence of the } \\
\text { task (46.4\%), } \\
\text { Social } \\
\text { interaction } \\
(33 \%) \text { and lack } \\
\text { of } \\
\text { accomplishment } \\
(26.8 \%)\end{array}$ \\
\hline $\begin{array}{l}\text { A12 } \\
\text { REDALYC }\end{array}$ & $\begin{array}{l}\text { Psychosocial risk } \\
\text { factors in the } \\
\text { workplace affect } \\
\text { teachers' mental } \\
\text { health depending on } \\
\text { the type of facility's } \\
\text { funding }\end{array}$ & $\begin{array}{l}\text { Caceres, } \\
\text { Campillay, } \\
\text { Cvitanic and } \\
\text { Bargsted } \\
\text { (2015). } \\
\text { Chile }\end{array}$ & $\begin{array}{l}\text { Psychological } \\
\text { requirements } \\
\text { (53.31\%), } \\
\text { Double } \\
\text { presence } \\
(48.36 \%) \text { and } \\
\text { Active work and } \\
\text { development } \\
\text { possibilities } \\
(33.15 \%) .\end{array}$ \\
\hline $\begin{array}{l}\text { A13 } \\
\text { PROQUEST }\end{array}$ & $\begin{array}{lr}\text { Psychosocial } & \text { risks, } \\
\text { occupational } & \text { stress } \\
\text { and } & \text { burnout } \\
\text { syndrome } & \text { in } \\
\text { university workers at } \\
\text { a bioanalysis school }\end{array}$ & $\begin{array}{l}\text { Seijas-Solano } \\
(2019) . \\
\text { Venezuela }\end{array}$ & $\begin{array}{l}\text { Work rhythm } \\
(80.8 \%), \\
\text { Insecurity in } \\
\text { working } \\
\text { conditions } \\
(54.9 \%) \text {, Work } \\
\text { stress }(3 \%) \text { and } \\
\text { burnout } \\
\text { syndrome (5\%) } \\
\text { in the most } \\
\text { unfavorable } \\
\text { situation. } \\
\text { Double } \\
\text { presence } \\
(52.7 \%) \text { and } \\
\text { Role conflict } \\
(38.9 \%), \\
\text { intermediate } \\
\text { situation. }\end{array}$ \\
\hline $\begin{array}{l}\text { A14 } \\
\text { PROQUEST }\end{array}$ & $\begin{array}{l}\text { The double presence } \\
\text { in female workers: } \\
\text { balance between } \\
\text { work and family life }\end{array}$ & 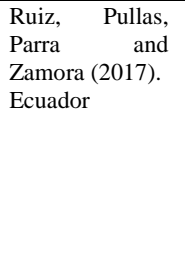 & $\begin{array}{l}\text { Double } \\
\text { presence } \\
(54.25 \%), \\
\text { quantitative } \\
\text { requirements } \\
(45.78 \%) \text { and } \\
\text { sensory } \\
\text { requirements } \\
(74.39 \%) .\end{array}$ \\
\hline $\begin{array}{l}\text { A15 } \\
\text { PROQUEST }\end{array}$ & $\begin{array}{l}\text { Prevalencer of } \\
\text { Burnout Syndrome in } \\
\text { teachers: Factors } \\
\text { associated with the } \\
\text { labor bonding statute } \\
\text { in Colombia }\end{array}$ & $\begin{array}{l}\text { Posada, } \\
\text { Molano, Parra, } \\
\text { Brito and Rubio } \\
\text { (2019). } \\
\text { Colombia }\end{array}$ & $\begin{array}{l}\text { Working } \\
\text { overtime, lack } \\
\text { of } \\
\text { communication } \\
\text { spaces with the } \\
\text { bosses, } \\
\text { inadequate } \\
\text { salary and lack } \\
\text { of support from } \\
\text { the bosses. }\end{array}$ \\
\hline $\begin{array}{l}\text { A16 } \\
\text { REDALYC }\end{array}$ & $\begin{array}{l}\text { Psychosocial, } \\
\text { burnout } \\
\text { psychosomatic risks } \\
\text { in public sector } \\
\text { workers }\end{array}$ & $\begin{array}{l}\text { Uribe-Prado } \\
(2020) \text {. } \\
\text { Mexico }\end{array}$ & $\begin{array}{l}\text { Performance } \\
\text { recognition } \\
\text { deficiente } \\
(26.9 \%), \text { pain } \\
\text { disorders } \\
(26.2 \%), \\
\text { workplace } \\
\text { violence } \\
(24.9 \%), \text { long } \\
\text { hours, adverse } \\
\text { leadership and } \\
\text { work } \\
\text { relationships } \\
\text { deficiente } \\
(24.6 \%)\end{array}$ \\
\hline $\begin{array}{l}\text { A17 } \\
\text { SCIELO }\end{array}$ & $\begin{array}{l}\text { Psychosocial risk } \\
\text { factors and perceived } \\
\text { stress in workers of } \\
\text { an electricity } \\
\text { company in Chile }\end{array}$ & $\begin{array}{l}\text { Güilgüiruca, } \\
\text { Meza, Góngora } \\
\text { and Moya } \\
\text { (2015). } \\
\text { Chile }\end{array}$ & $\begin{array}{l}\text { Social support } \\
(34.2 \%) \text {, } \\
\text { psychological } \\
\text { demands } \\
(32.2 \%) \text {, active } \\
\text { work (31.5\%), } \\
\text { compensation } \\
(34.2 \%) \text { and } \\
\text { double presence } \\
(44 \%) \text {. }\end{array}$ \\
\hline
\end{tabular}

\begin{tabular}{|c|c|c|c|}
\hline $\begin{array}{l}\text { Code/ } \\
\text { Base }\end{array}$ & Title & $\begin{array}{l}\text { Author/ } \\
\text { Country }\end{array}$ & $\begin{array}{l}\text { Main FRP } \\
\text { found }\end{array}$ \\
\hline $\begin{array}{l}\text { A18 } \\
\text { SCIELO }\end{array}$ & $\begin{array}{l}\text { Teaching stress and } \\
\text { psychosocial factors } \\
\text { in teachers in Latin } \\
\text { America, North } \\
\text { America and Europe }\end{array}$ & $\begin{array}{l}\text { Alvites- } \\
\text { Huamaní } \\
(2019) . \\
\text { Peru }\end{array}$ & $\begin{array}{l}\text { There is a } \\
\text { significant } \\
\text { correlation } \\
\text { between teacher } \\
\text { stress and } \\
\text { psychosocial } \\
\text { factors, with the } \\
\text { level of stress } \\
\text { due to anxiety, } \\
\text { depression and } \\
\text { maladaptive } \\
\text { beliefs } \\
\text { prevailing. }\end{array}$ \\
\hline $\begin{array}{l}\text { A19 } \\
\text { SCIELO }\end{array}$ & $\begin{array}{lr}\text { Psychosocial factors } \\
\text { and } & \text { Burnout } \\
\text { syndrome r in } \\
\text { academics from a } \\
\text { public university in } \\
\text { Mexico }\end{array}$ & $\begin{array}{l}\text { Villamar, } \\
\text { Juárez, } \\
\text { González and } \\
\text { Osnaya (2019). } \\
\text { Mexico }\end{array}$ & $\begin{array}{l}\text { Social problems } \\
(40.9 \%), \\
\text { Organizational } \\
\text { problems } \\
(34.8 \%), \\
\text { Burnout } \\
(33.6 \%)\end{array}$ \\
\hline $\begin{array}{l}\text { A20 } \\
\text { DIALNET }\end{array}$ & $\begin{array}{l}\text { Psychosocial factors } \\
\text { that influence work } \\
\text { behavior according } \\
\text { to the processes of } \\
\text { administrative } \\
\text { management and } \\
\text { human talent } \\
\text { presented by } \\
\text { employees of the } \\
\text { company Distraves } \\
\text { S.A.de Cúcuta }\end{array}$ & $\begin{array}{l}\text { Jaimes, } \\
\text { Márquez and } \\
\text { Pernía (2015). } \\
\text { Colombia }\end{array}$ & $\begin{array}{l}\text { Lack } \\
\text { training; } \\
\text { conditions in } \\
\text { their jobs and } \\
\text { availability of } \\
\text { resources. }\end{array}$ \\
\hline $\begin{array}{l}\text { A21 } \\
\text { PROQUEST }\end{array}$ & $\begin{array}{l}\text { Psychosocial factors } \\
\text { and psychic wear and } \\
\text { tear in the work } \\
\text { environment }\end{array}$ & $\begin{array}{l}\text { Carrión-García, } \\
\text { Hernández- } \\
\text { Gracia (2017). } \\
\text { Mexico }\end{array}$ & $\begin{array}{l}\text { Harmful } \\
\text { Working } \\
\text { Conditions in } \\
\text { Working } \\
\text { Context } \\
(13.6 \%) \text {, } \\
\text { Working } \\
\text { Content } \\
(22.1 \%) \text {, } \\
\text { Individual } \\
\text { Factors (11.4\%) } \\
\text { and Perceived } \\
\text { Psychic Wear } \\
(11.4 \%)\end{array}$ \\
\hline
\end{tabular}

Table 1 Description of the articles selected for the systematic review

$100 \%$ of the studies have a quantitative approach; however, there is a variation in the methodological aspects to assess psychosocial risk factors, where $66.7 \%$ is descriptive, $52.4 \%$ non-experimental, and $76.2 \%$ cross-sectional (Table 2).

The number of participants in the research ranged from 16 to 597, and included a total of 3,608 workers from companies in Latin American countries.

Regarding the sociodemographic data, only four investigations (19\%) did not report the gender of the participants (studies A3, A15, A18 and A20), while in seven studies the sample of the male gender was larger (33.3\%), and in 10 studies, the female gender sample prevailed $(47.6 \%)$.
SUÁREZ-REYES, Susana, AGUILAR-MORALES, Norma and MAGAÑA-MEDINA, Deneb Elí. Instruments to identify psychosocial risk factors at work: a systematic review. Journal- Labor and Demographic economic. 2020 
Most of the studies were conducted in Mexico (38\%), followed by Colombia (24\%), Peru (14\%), Chile (14\%), Ecuador (5\%) and Venezuela (5\%). Of the selected investigations, the sector in which more inquiries have been made about psychosocial risk factors is education $(29 \%)$, followed by the health sector (24\%), while the energy sector (5\%) and services $(5 \%)$ are poorly studied.

\begin{tabular}{|c|c|c|c|c|c|}
\hline Code. & Sample & Sector & Design & $\% \mathrm{H}$ & $\% \mathbf{M}$ \\
\hline $\mathrm{A} 1$ & 150 workers & Industrial & $\begin{array}{l}\text { Descriptive, } \\
\text { transversal }\end{array}$ & $68 \%$ & $32 \%$ \\
\hline $\mathrm{A} 2$ & $\begin{array}{l}50 \text { technicians, } \\
\text { assistants and } \\
\text { operators }\end{array}$ & Services & 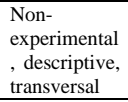 & $80 \%$ & $20 \%$ \\
\hline $\mathrm{A} 3$ & 183 workers & Sanitary & $\begin{array}{l}\text { Non- } \\
\text { experimental } \\
\text {, descriptive, } \\
\text { transversal }\end{array}$ & - & - \\
\hline A4 & $\begin{array}{l}142 \text { workers in } \\
\text { health centers and } \\
\text { posts }\end{array}$ & Sanitary & $\begin{array}{l}\text { Non- } \\
\text { experimental } \\
\text {, descriptive, } \\
\text { cross- } \\
\text { sectional, } \\
\text { relational }\end{array}$ & - & $100 \%$ \\
\hline A5 & $\begin{array}{l}121 \text { workers from } \\
\text { a government } \\
\text { institution }\end{array}$ & $\begin{array}{l}\text { Of the } \\
\text { administratio } \\
n\end{array}$ & $\begin{array}{l}\begin{array}{l}\text { Non- } \\
\text { experimental }\end{array} \\
\text { correlational } \\
\text { Sectional }\end{array}$ & $44 \%$ & $56 \%$ \\
\hline A6 & $\begin{array}{l}117 \text { university } \\
\text { teachers }\end{array}$ & Educational & $\begin{array}{l}\text { Correlational } \\
\text { and } \\
\text { transversal }\end{array}$ & $52 \%$ & $48 \%$ \\
\hline A7 & $\begin{array}{l}106 \text { workers of a } \\
\text { fruit trading and } \\
\text { exporting } \\
\text { company }\end{array}$ & Commercial & $\begin{array}{l}\text { Observation } \\
\text { al, } \\
\text { transversal }\end{array}$ & - & $100 \%$ \\
\hline A8 & $\begin{array}{l}199 \text { workers in a } \\
\text { manufacturing } \\
\text { industry. }\end{array}$ & $\begin{array}{l}\text { Industrial } \\
\end{array}$ & 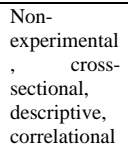 & $39.2 \%$ & $60.8 \%$ \\
\hline A9 & $\begin{array}{l}16 \text { nurses and } 64 \\
\text { nursing assistants }\end{array}$ & Sanitary & Descriptive & $10 \%$ & $90 \%$ \\
\hline A10 & 357 nurses & Sanitary & $\begin{array}{l}\text { Cross- } \\
\text { sectional, } \\
\text { observationa } \\
1, \text { analytical }\end{array}$ & $2 \%$ & $98 \%$ \\
\hline A11 & $\begin{array}{l}97 \quad \text { general } \\
\text { practitioners }\end{array}$ & Sanitary & $\begin{array}{l}\text { Cross- } \\
\text { sectional, } \\
\text { analytical } \\
\end{array}$ & $50.5 \%$ & $49.5 \%$ \\
\hline A12 & 340 teachers & Educational & 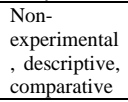 & $40 \%$ & $60 \%$ \\
\hline A13 & $\begin{array}{l}75 \quad \text { university } \\
\text { professors }\end{array}$ & Educational & $\begin{array}{l}\text { Descriptive, } \\
\text { transversal }\end{array}$ & $14.7 \%$ & $85.3 \%$ \\
\hline A14 & $\begin{array}{l}67 \text { employees of a } \\
\text { textile company }\end{array}$ & Industrial & $\begin{array}{l}\text { Descriptive, } \\
\text { transversal }\end{array}$ & $32.8 \%$ & $65.7 \%$ \\
\hline A15 & $\begin{array}{l}54 \text { teachers from } \\
\text { an Educational } \\
\text { Institution }\end{array}$ & Educational & $\begin{array}{l}\text { Correlational } \\
\text {, descriptive }\end{array}$ & - & - \\
\hline A16 & $\begin{array}{lr}305 & \text { Mexican } \\
\text { workers } & \text { of a } \\
\text { human } & \text { rights } \\
\text { institution } & \end{array}$ & $\begin{array}{l}\text { Of the } \\
\text { administratio } \\
n\end{array}$ & $\begin{array}{l}\text { Descriptive, } \\
\text { Pearson } \\
\text { correlation } \\
\text { analysis, } \\
\text { structural } \\
\text { equations. }\end{array}$ & $50.8 \%$ & $49.2 \%$ \\
\hline A17 & 292 workers & Energetic & $\begin{array}{l}\text { Non- } \\
\text { experimental } \\
\text { descriptive, } \\
\text { correlational }\end{array}$ & $82.5 \%$ & $17.5 \%$ \\
\hline A18 & $\begin{array}{lr}81 \text { basic and higher } \\
\text { education } \\
\text { fromeachers } \\
\begin{array}{l}\text { Americar, Latin } \\
\text { America }\end{array} \\
\begin{array}{l}\text { Europe } \\
\text { Eurth }\end{array} \\
\end{array}$ & Educational & 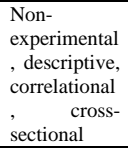 & - & - \\
\hline A19 & $\begin{array}{l}247 \text { professors and } \\
\text { researchers }\end{array}$ & Educational & $\begin{array}{l}\text { Non- } \\
\text { experimental } \\
, \quad \text { cross- } \\
\text { sectional, } \\
\text { correlational }\end{array}$ & $49.4 \%$ & $50.6 \%$ \\
\hline A20 & $\begin{array}{l}12 \text { employees of } \\
\text { the company } \\
\text { Distraves S.A. }\end{array}$ & Commercial & $\begin{array}{l}\text { Non- } \\
\text { experimental } \\
\text { descriptive, } \\
\text { transversal }\end{array}$ & - & - \\
\hline A21 & $\begin{array}{l}597 \text { workers of a } \\
\text { company in the } \\
\text { industrial sector in } \\
\text { Jalisco (Mexico) }\end{array}$ & Industrial & 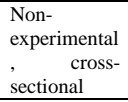 & $57.8 \%$ & $37.4 \%$ \\
\hline
\end{tabular}

Table 2 Description of the gender data, sample and design of the selected studies
The instruments for data collection varied depending on the objective and size of the sample, where 20 scales were identified to identify and evaluate the psychosocial risk factors (Table 3 ).

According to the data, the most widely implemented instrument is the ISTAS21, in its short and medium version $(38.1 \%)$, which was designed by the Trade Union Institute of Work, Environment and Health (2002), and has 5 dimensions, with each of them.

Likewise, the Battery of instruments for the evaluation of psychosocial risk factors was implemented in $14.3 \%$ of the selected studies, an instrument designed by the Pontificia Universidad Javeriana and the Ministry of Social Protection of Colombia, which has different questionnaires to identify said factors (Ministry of Social Protection, 2010).

\begin{tabular}{|c|c|c|}
\hline Code. & Instrument & Valuated dimensions \\
\hline A1 & $\begin{array}{ll}- & \text { Symptomatic } \\
\text { Stress Scale } \\
\text { Scale } \\
\text { Psychosocial of } \\
\text { Factors at Work }\end{array}$ & $\begin{array}{l}18 \text { items of symptoms associated } \\
\text { with stress (somatic, } \\
\text { physiological or emotional } \\
\text { nature). } \\
\text { Conditions of the workplace, } \\
\text { Workload, Content and } \\
\text { characteristics of the task, Labor } \\
\text { demands, Labor role and career } \\
\text { development, Social interaction } \\
\text { and organizational aspects and } \\
\text { Performance compensation. }\end{array}$ \\
\hline A2 & $\begin{array}{l}\text { Battery of } \\
\text { instruments for } \\
\text { the evaluation } \\
\text { of psychosocial } \\
\text { risk factors } \\
\text { (Ministry of } \\
\text { Labor and the } \\
\text { Javeriana } \\
\text { University): } \\
\text { Questionnaire } \\
\text { of intra-labor } \\
\text { psychosocial } \\
\text { risk factors } \\
\text { (form B) }\end{array}$ & $\begin{array}{l}\text { Work demands (Quantitative } \\
\text { demands, Mental load demands, } \\
\text { Emotional demands, Demands of } \\
\text { responsibility of the position, } \\
\text { Environmental and physical } \\
\text { effort demands, Demands of the } \\
\text { working day, Consistency of the } \\
\text { role Influence of the work } \\
\text { environment on the extra work). } \\
\text { Control (Control and autonomy } \\
\text { over work, Opportunities for } \\
\text { development and use of abilities } \\
\text { and skills, Participation and } \\
\text { change management, Clarity of } \\
\text { role, Training). } \\
\text { Leadership and social relations at } \\
\text { work (Characteristics of } \\
\text { leadership, Social relations at } \\
\text { work, Performance feedback, } \\
\text { Relationship with collaborators). } \\
\text { Reward (Recognition and } \\
\text { compensation Rewards derived } \\
\text { from belonging to the } \\
\text { organization and the work that is } \\
\text { done). }\end{array}$ \\
\hline A3 & $\begin{array}{l}\text { Battery of } \\
\text { instruments for } \\
\text { the evaluation } \\
\text { of psychosocial } \\
\text { risk factors } \\
\text { (Ministry of } \\
\text { Labor and the } \\
\text { Javeriana } \\
\text { University): } \\
\text { Questionnaire } \\
\text { on intra- } \\
\text { occupational } \\
\text { psychosocial } \\
\text { risk factors } \\
\text { (form B) and the } \\
\text { Questionnaire } \\
\text { on Non- } \\
\text { occupational } \\
\text { Psychosocial } \\
\text { Risk Factors. }\end{array}$ & $\begin{array}{l}\text { Form B: Demands of work, } \\
\text { Control, Leadership and social } \\
\text { relations at work and Reward, } \\
\text { described in publication A2. } \\
\text { Non-work questionnaire: Time } \\
\text { away from work, Family } \\
\text { relationships, Communication } \\
\text { and interpersonal relationships, } \\
\text { Economic situation of the family } \\
\text { group, Characteristics of the } \\
\text { home and its environment, } \\
\text { Influence of the non-work } \\
\text { environment on work and Home- } \\
\text { work-home movement. }\end{array}$ \\
\hline
\end{tabular}

SUÁREZ-REYES, Susana, AGUILAR-MORALES, Norma and MAGAÑA-MEDINA, Deneb Elí. Instruments to identify psychosocial risk factors at work: a systematic review. Journal- Labor and Demographic economic. 2020 


\begin{tabular}{|c|c|c|}
\hline Code. & Instrument & Valuated dimensions \\
\hline $\mathrm{A} 4$ & ISTAS 21 & $\begin{array}{l}\text { Psychological demands } \\
\text { (cognitive demands, quantitative } \\
\text { demands, sensory demands, } \\
\text { emotional demands and demands } \\
\text { to hide emotions). } \\
\text { Active work (Development } \\
\text { possibilities, Integration in the } \\
\text { company, Control over working } \\
\text { time, Sense of work, Influence on } \\
\text { general work conditions and } \\
\text { Influence on specific work } \\
\text { conditions). } \\
\text { Social support in the company } \\
\text { (Clarity of role, Conflict of role, } \\
\text { Quality of leadership, } \\
\text { Possibilities of social } \\
\text { relationship, Quality of } \\
\text { relationship with superiors and } \\
\text { Quality of relationship with } \\
\text { colleagues). } \\
\text { Compensations (Estimates, } \\
\text { Insecurity regarding the } \\
\text { conditions of the contract and } \\
\text { Insecurity regarding the } \\
\text { conditions of the work } \\
\text { performed). } \\
\text { Double presence (Concerns about } \\
\text { housework, Burden of } \\
\text { housework). }\end{array}$ \\
\hline A5 & $\begin{array}{l}\text { FRP: (NOM-035- } \\
\text { STPS) (Campa, } \\
\text { 2018), in the } \\
\text { revised version by } \\
\text { Almirall, et al. } \\
\text { (2018). } \\
\text { Shackelton's } \\
\text { Reward-Effort } \\
\text { Imbalance } \\
\text { Questionnaire, } \\
\text { Spanish Version }\end{array}$ & $\begin{array}{l}\text { Conditions in the work } \\
\text { environment, Workload, Lack of } \\
\text { control over work, Working } \\
\text { hours, Interference in the work- } \\
\text { family relationship, Leadership, } \\
\text { Work relationships and Violence. } \\
\text { Extrinsic effort and reward. }\end{array}$ \\
\hline A6 & $\begin{array}{llr}\text { - } & \text { ISTAS } 21 & \text { short } \\
\text { version. } & \\
\text { - } & \text { PSS14 Perceived } \\
& \text { Stress } & \text { Scale, } \\
& \text { Mexican } & \\
\text { adaptation } & \end{array}$ & $\begin{array}{l}\text { Dimensions mentioned in } \\
\text { publication A4. } \\
\text { Perceived stress, Family and } \\
\text { friend support, Hamilton } \\
\text { Depression, } \\
\text { symptomatology and Violence } \\
\text { and severity index. }\end{array}$ \\
\hline A7 & $\begin{array}{ll}- & \text { ISTAS21 } \\
- & \text { Questionnaire } \\
\text { S10/12 }\end{array}$ & $\begin{array}{l}\text { Dimensions mentioned in } \\
\text { publication A4. } \\
\text { Satisfaction with the benefits } \\
\text { received, Satisfaction with the } \\
\text { physical environment of the } \\
\text { company and Satisfaction with } \\
\text { supervision. }\end{array}$ \\
\hline A8 & $\begin{array}{l}\text { Battery for the } \\
\text { study } \\
\text { Psychosocial of } \\
\text { Conditions of } \\
\text { Work (CTCPS- } \\
\text { MAC), validated } \\
\text { in a Latin } \\
\text { American } \\
\text { population } \\
\text { (Colombia, } \\
\text { Ecuador, Mexico, } \\
\text { Peru and } \\
\text { Venezuela) by a } \\
\text { group of experts }\end{array}$ & $\begin{array}{l}\text { - Work Context (Culture of the } \\
\text { organization and management, } \\
\text { Role or role in the organization, } \\
\text { Interrelation of work with family } \\
\text { and social problems and } \\
\text { Interpersonal relations at work). } \\
\text { Work Content (Workload and } \\
\text { rhythm, Work environments, } \\
\text { Equipment and physical agents, } \\
\text { and Conceptions of job tasks). } \\
\text { Individual Factors (Psychic risk } \\
\text { buffers, Company characteristics } \\
\text { and Personal characteristics). } \\
\text { Psychic Burnout (Subjective } \\
\text { symptoms and health disorders - } \\
\text { Psychological states- Emotional } \\
\text { cognitive response, Subjective } \\
\text { symptoms and health disorders - } \\
\text { Psychological states- Behavioral } \\
\text { response and Subjective } \\
\text { symptoms and health disorders - } \\
\text { Psychological states- } \\
\text { Physiological response). }\end{array}$ \\
\hline A9 & $\begin{array}{l}\text { Diagnosis of } \\
\text { Psychosocial } \\
\text { Factors }\end{array}$ & $\begin{array}{l}\text { Content of the task, Human } \\
\text { relations, Organization of work } \\
\text { time and Personnel management. }\end{array}$ \\
\hline A10 & $\begin{array}{ll}- & \text { Perceived Stress } \\
\text { Scale } \\
\text { Job Content } \\
\text { Questionnaire } \\
\text { Questionnaire for } \\
\text { the Evaluation of } \\
\text { Burn-Out } \\
\text { Syndrome }\end{array}$ & $\begin{array}{l}\text { Favor of control and loss of } \\
\text { control. } \\
\text { Psychological demands, Use of } \\
\text { skills, Decision authority, Social } \\
\text { support from colleagues and } \\
\text { Social support from the boss. } \\
\text { Illusion for work, mental } \\
\text { exhaustion, indolence and guilt. }\end{array}$ \\
\hline
\end{tabular}

\begin{tabular}{|c|c|c|}
\hline Code. & Instrument & Valuated dimensions \\
\hline A11 & $\begin{array}{l}\text { Guide for the } \\
\text { identification of } \\
\text { psychosocial } \\
\text { factors of the } \\
\text { Mexican Institute } \\
\text { of Social Security } \\
\text { Scale of "Maslach } \\
\text { Burnout } \\
\text { Inventory (MBI)" }\end{array}$ & $\begin{array}{l}\text { Emotional exhaustion, } \\
\text { depersonalization and decreased } \\
\text { personal fulfillment at work. }\end{array}$ \\
\hline A12 & $\begin{array}{ll}- & \text { ISTAS 21 } \\
- & \text { DASS 21 } \\
- & \text { MBI scale }\end{array}$ & $\begin{array}{ll}- & \text { Dimensions mentioned in } \\
\text { publication A4. } & \text { Depression, anxiety and stress. } \\
- & \text { Emotional exhaustion, } \\
\text { depersonalization and decreased } \\
\text { personal fulfillment at work. }\end{array}$ \\
\hline A13 & $\begin{array}{ll}\text { ISTAS21, } \\
\text { medium versión }\end{array}$ & $\begin{array}{ll}\text { Dimensions mentioned in } \\
\text { publication A4. }\end{array}$ \\
\hline A14 & $\begin{array}{ll}- & \begin{array}{l}\text { ISTAS21, short } \\
\text { versión }\end{array} \\
\end{array}$ & $\begin{array}{llr}- & \begin{array}{l}\text { Dimensions mentioned } \\
\text { publication A4. }\end{array}\end{array}$ \\
\hline A15 & $\begin{array}{l}\text { Battery of } \\
\text { instruments for } \\
\text { the evaluation of } \\
\text { psychosocial risk } \\
\text { factors (Ministry } \\
\text { of Labor and the } \\
\text { Javeriana } \\
\text { University). }\end{array}$ & $\begin{array}{l}\text { Communication with superiors, } \\
\text { Sufficient salary, Working } \\
\text { overtime, Social recognition, } \\
\text { Absences and penalties, Support } \\
\text { from superiors, Pauses during the } \\
\text { day, Work evaluation, Tasks } \\
\text { according to the teaching } \\
\text { profession, Relationship with } \\
\text { colleagues, Working hours, } \\
\text { Union membership and } \\
\text { Opportunities training. }\end{array}$ \\
\hline A16 & 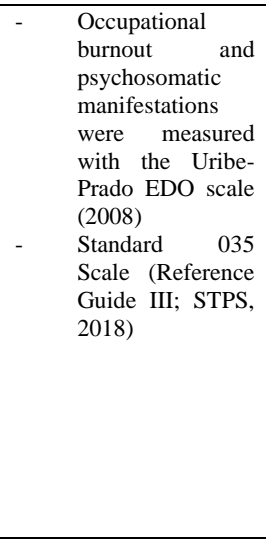 & $\begin{array}{l}\text { Emotional exhaustion, } \\
\text { Depersonalization, Achievement } \\
\text { dissatisfaction, Sleep disorders, } \\
\text { Pain disorders, Gastrointestinal } \\
\text { disorders, Psychoneurotic } \\
\text { disorders, Anxiety indicator and } \\
\text { Depression indicator. } \\
\text { Recognition of poor performance, } \\
\text { Work violence, Long working } \\
\text { hours, Pain disorders, Emotional } \\
\text { burnout, Adverse leadership, } \\
\text { Poor work relationships, Work- } \\
\text { family interference, Sleep } \\
\text { disorders, Anxiety indicator, } \\
\text { Achievement dissatisfaction, } \\
\text { Depression indicator, Disorders } \\
\text { Gastrointestinal, } \\
\text { Depersonalization } \\
\text { Psychoneurotic Disorders. and } \\
\end{array}$ \\
\hline A17 & $\begin{array}{lll}- & \text { ISTAS21, } & \text { short } \\
& \text { version } & \\
- & \text { Perceived } & \text { Stress } \\
& \text { Scale } & \end{array}$ & $\begin{array}{ll}\text { - } & \text { Dimensions mentioned in } \\
\text { publication A4. } \\
\text { Unpredictability, } \\
\text { Uncontrollability, Overload, and } \\
\text { direct questions about } \\
\text { experienced stress levels. }\end{array}$ \\
\hline A18 & $\begin{array}{l}\text { Questionnaire on } \\
\text { Psychosocial } \\
\text { Factors in } \\
\text { Academic Work }\end{array}$ & $\begin{array}{l}\text { Workplace conditions, Workload, } \\
\text { Content and characteristics of the } \\
\text { task, Labor demands, Academic } \\
\text { role and career development, } \\
\text { Social interaction and } \\
\text { organizational aspects and } \\
\text { Performance compensation. }\end{array}$ \\
\hline A19 & $\begin{array}{l}\text { Online battery } \\
\text { adapted from the } \\
\text { questionnaire on } \\
\text { Psychosocial } \\
\text { Factors of } \\
\text { Academic Work } \\
\text { (FPSIS) } \\
\text { Questionnaire for } \\
\text { the Evaluation of } \\
\text { Burn-Out } \\
\text { Syndrome for } \\
\text { Education } \\
\text { Professionals } \\
\text { (CESQT-PE) } \\
\end{array}$ & $\begin{array}{l}\text { Social and organizational } \\
\text { problems, Excessive working } \\
\text { hours, Insufficient spaces and } \\
\text { materials, Problems with } \\
\text { students, Dissatisfaction with } \\
\text { financial reward, Mental and } \\
\text { physical effort and Biochemical } \\
\text { risks. } \\
\text { Illusion for work, Psychic } \\
\text { exhaustion, Indolence, Guilt. }\end{array}$ \\
\hline A20 & ISTAS 21 & $\begin{array}{llr}- & \begin{array}{l}\text { Dimensions mentioned } \\
\text { publication A4. }\end{array} & \text { in } \\
\end{array}$ \\
\hline A21 & $\begin{array}{l}\text { Battery for the } \\
\text { study } \\
\text { psychosocial of } \\
\text { working } \\
\text { conditions } \\
\text { CTCPS-MAC }\end{array}$ & $\begin{array}{l}\text { Dimensions mentioned in study } \\
\text { A8. }\end{array}$ \\
\hline
\end{tabular}

Table 3 Scales to identify and evaluate Psychosocial Risk Factors in selected studies
SUÁREZ-REYES, Susana, AGUILAR-MORALES, Norma and MAGAÑA-MEDINA, Deneb Elí. Instruments to identify psychosocial risk factors at work: a systematic review. Journal- Labor and Demographic economic. 2020 


\section{Conclusions}

In organizations, people are the most important resource; However, they are exposed to different risks that generate health problems, especially those that damage their mental health, known as psychosocial risk factors, so it is necessary to incorporate processes and strategies that promote their physical and mental well-being. through the implementation of instruments that allow identifying and evaluating the influence of these factors, in order to eliminate or reduce them (Muñoz, Orellano and Hernández, 2018).

The review and analysis carried out in this work have made it possible to know the different scales that have been designed to identify and evaluate the psychosocial risk factors at work, where in recent years their study has become more relevant, since norms, laws have been implemented and regulations to regulate and oblige companies to analyze and evaluate these factors.

However, one of the findings found in this research revealed that the identification and evaluation of psychosocial risk factors is still deficient, since so far there are no relevant investigations in many of the Latin American countries, since they have not been detected in the search, and most of the articles excluded were for having a merely theoretical approach, so the corresponding authorities should raise awareness about the importance of addressing these risks in a timely manner before they negatively influence workers .

Although there are different scales for the study of these factors, the best known and implemented are the ISTAS21 $(38.1 \%)$ and the Battery of instruments for the evaluation of psychosocial risk factors (14.3\%), which are versions that can be applied in Spanish-speaking countries; However, it does not mean that they are adequate to apply in all investigations, since there is a variety of instruments that can be chosen, depending on the factors to be investigated.
The review of the selected studies confirms the presence of psychosocial risk factors in workers from different sectors of Latin American companies, prevailing factors of double presence (33.3\%), psychosocial demands $(28.6 \%)$, as well as the conditions $(23.8 \%)$ and workload (19\%), which can be reflected not only in work errors, but also in work accidents, in the development of work stress and in diseases that can even lead to death (Moreno and Báez, 2010). Psychosocial risk factors generate negative impacts on personnel regardless of gender, also influencing their quality of life, as well as the productive conditions of the organization, so it is important to ensure the physical and mental well-being of workers (Cobo , Muñoz, Martos, Carmona, Pérez, Cirici and García-Parés, 2010). Ultimately, such risks must be prevented and healthy environments created, taking into account work interactions, organizational conditions and other factors that influence the performance of workers, in such a way that productivity and growth of the company can be guaranteed.

\section{References}

Agencia Europea para la Seguridad y la Salud en el Trabajo (EU-OSHA). (2020). Los riesgos psicosociales y el estrés en el trabajo. Recuperado de: https://osha.europa.eu/es/themes/psychosocialrisk-and-stress

Alvites-Huamaní, C. G. (2019). Estrés docente y factores psicosociales en docentes de Latinoamérica, Norteamérica y Europa. Propósitos y Representaciones, 7 (3), 141-178. Recuperado de http://www.scielo.org.pe/pdf/pyr/v7n3/a06v7n3 .pdf

Aranda, C., Barraza, J. H., Romero, J. J., Quiñonez, L. A., Ceniceros, A. P., González, G. y Esparza, J. A. (2015). Factores psicosociales y síndrome de Burnout en médicos generales de Tepic, Nayarit (México). Salud Uninorte, 31 (2), 245-254. Recuperado de https://www.proquest.com/docview/173175393 6/C8EE931C23E64262PQ/1 
Aranda, C., Mares, F. D., Ramírez, B. Y. y Rojas, N. (2016). Factores psicosociales y síntomas de estrés laboral en trabajadores del área de producción de una empresa metalmecánica de El Salto, Jalisco. Cuadernos Hispanoamericanos de Psicología, 16 (2), 5-32. Recuperado de https://dialnet.unirioja.es/servlet/articulo?codig o=5969552

Bobadilla, C. A., Garzón, L. M. y Charria, V. H. (2018). Identificación de factores de riesgo psicosocial en una clínica de alta complejidad. Psicología desde el Caribe, 35 (2), 131-144. Recuperado de https://www.proquest.com/docview/228524255 8/DAA4381972024B80PQ/1

Brito-Ortíz, J. F., Juárez-García, A., NavaGómez, M. E., Castillo-Pérez, J. J. y Brito-Nava, E. (2019). Factores psicosociales, estrés psicológico y burnout en enfermería: un modelo de trayectorias. Enfermería Universitaria, 16 (2), 138-148. Recuperado de http://dx.doi.org/10.22201/eneo.23958421e.201 9.2.634

Bustillo-Guzmán, Rojas-Meriño, SánchezCamacho, Sánchez-Puello, Montalvo-Prieto y Rojas-López (2015). Riesgo psicosocial en el personal de enfermería. Servicio de urgencias en hospital universitario de Cartagena. Revista Duazary, 12 (1), 32-40. Recuperado de https://www.proquest.com/docview/183569289 0/6C2567D67A694BA7PQ/1

Caceres, N., Campillay, J., Cvitanic, C. y Bargsted, M. (2015). Los factores de riesgo psicosocial del trabajo afectan la salud mental de los profesores según el tipo de financiamiento del establecimiento. Salud y Sociedad, 6 (1), 5075. Recuperado de http://www.redalyc.org/articulo.oa?id=4397424 77004

Carrión-García, M. A. y Hernández-Gracia, T. J. (2017). Factores psicosociales y desgaste psíquico en ámbito laboral. Salud Uninorte, 34 (3), 705-714. Recuperado de https://www.proquest.com/docview/226010497 1/E02EB2A854E349E3PQ/1
Cobo, J., Muñoz, R., Martos, A., Carmona, M., Pérez, M., Cirici, R., y García-Parés, G. (2010). La violencia de género en la atención psiquiátrica y psicológica especializada: ¿es relevante la violencia de género para nuestros profesionales? Revista de Psiquiatría y Salud Mental, 3 (2), 61-67. Recuperado de https://www.elsevier.es/es-revista-revistapsiquiatria-salud-mental--286-pdfS1888989110000054

Comité Mixto OIT/OMS (1984). Factores psicosociales del trabajo: naturaleza, incidencia y prevención. Informe del comité mixto de la Organización Internacional del Trabajo y la Organización Mundial de la Salud sobre medicina del trabajo. Ginebra: Suiza. Recuperado http://www.factorespsicosociales.com/wpcontent/uploads/2019/02/FPS-OIT-OMS.pdf

Gil-Monte, P. R. (2012). Riesgos psicosociales en el trabajo y salud ocupacional. Revista Peruana Médica Experimental y Salud Publica, 29 (2), 237-241. Recuperado de http://www.scielo.org.pe/pdf/rins/v29n2/a12v29 n2.pdf

Gil-Monte, P. R., López-Vílchez, J., LlorcaRubio, J. L. y Sánchez-Piernas, J. (2016). Prevalencia de riesgos psicosociales en personal de la administración de justicia de la Comunidad Valenciana (España). Liberabit, 22 (1), 7-19. Recuperado de http://www.scielo.org.pe/pdf/liber/v22n1/a01v2 2n1.pdf

Güilgüiruca, M., Meza, K., Góngora, R. y Moya, C. (2015). Factores de riesgo psicosocial y estrés percibido en trabajadores de una empresa eléctrica en Chile. Medicina y Seguridad del Trabajo, 61 (238), 57-67. Recuperado de http://scielo.isciii.es/scielo.php?script=sci_artte xt\&pid=S0465-546X2015000100006

Henry, M. L. (2017). Hacia un enfoque ampliado de los riesgos laborales en Argentina El estudio de los riesgos psicosociales y su incidencia en el sector público. Cuadernos del Cendes, (96), 109-128. Recuperado de https://www.redalyc.org/pdf/403/40354944005. pdf

Instituto Nacional de Seguridad y Salud en el Trabajo [INSST], (s.f.). Riesgos Psicosociales. Recuperado de https://www.insst.es/riesgospsicosociales 2

SUÁREZ-REYES, Susana, AGUILAR-MORALES, Norma and MAGAÑA-MEDINA, Deneb Elí. Instruments to identify psychosocial risk factors at work: a systematic review. Journal- Labor and Demographic economic. 2020 
Jaimes, S. S., Márquez, J. V. y Pernía, L. P. (2015). Factores Psicosociales que Influyen en el Comportamiento Laboral de Acuerdo con los Procesos de Gestión Administrativa y del Talento Humano que Presentan los Empleados de la Empresa Distraves S.A de Cúcuta. Revista Mundo FESC, (9), 64-68. Recuperado de https://dialnet.unirioja.es/servlet/articulo?codig $\mathrm{o}=5286658$

Juárez-García, A. (2015). Investigaciones psicométricas de escalas psicosociales en trabajadores mexicanos. México: Plaza y Valdés Editores-UAEM. Recuperado de https://www.researchgate.net/publication/29776 3387_Investigaciones_psicometricas_de_escala s_psicosociales_en_trabajadores_mexicanos/lin k/56e3692a08aedb4cc8a859c5/download

Leka, S. y Jain, A. (2010). Health impact of psychosocial hazards at work: an overview. World Health Organization. Recuperado de https://apps.who.int/iris/handle/10665/44428

Luna-Chávez, E.A., Anaya-Velasco, A. y Ramírez-Lira, E. (2019). Diagnóstico de las percepciones de los factores de riesgo psicosociales en el trabajo del personal de una industria manufacturera. Estudios de Psicología (Campinas), (36), 1-10. http://dx.doi.org/10.1590/1982-

$0275201936 \mathrm{e} 180148$

Ministerio de la Protección Social. (2008). Resolución 2646 de 2008 Factores de riesgo psicosocial en el trabajo y determinación del origen de las patologías causadas por el estrés ocupacional. Recuperado de http://steel.net.co/resolucion-2646-de-2008/ Ministerio de la Protección Social. (2010). Batería de instrumentos para la evaluación de factores de riesgo psicosocial. Recuperado de https://portal.posipedia.co/wp-

content/uploads/2019/08/bateria-instrumento-

evaluacion-factores-riesgo-psicosocial.pdf

Moreno, B. (2011). Factores y riesgos laborales psicosociales: conceptualización, historia y cambios actuales. Medicina y Seguridad del Trabajo, 57, 4-19. Recuperado de http://scielo.isciii.es/pdf/mesetra/v57s1/especial .pdf
Moreno, B., y Báez, C. (2010). Factores y Riesgo Psicosociales, formas, consecuencias, medidas y buenas prácticas. Madrid: INSHTUAM. Recuperado de http://biblioteca.uoc.edu/es/recursos/recurso/fac tores-y-riesgos-psicosociales-formasconsecuencias-medidas-y-buenas-practicas

Muñoz, D., Orellano, N. y Hernández, H. (2018). Riesgo psicosocial: tendencias y nuevas orientaciones laborales. Psicogente, 21 (40), 532-544. Recuperado de https://www.proquest.com/docview/232959010 2/35E51358257745CCPQ/34

Muñoz, D., Pitre, P. y Amaya, N. (2017). Diagnóstico de los factores de riesgos psicosociales intralaborales en la empresa Miramar en el municipio de Maicao en la Guajira. Aglala, 8 (1), 243-264. Recuperado de https://dialnet.unirioja.es/servlet/articulo?codig $\mathrm{o}=6832755$

Neffa, J. C. (2015). Los riesgos psicosociales en el trabajo: contribución a su estudio. Buenos Aires: Universidad Nacional de La Plata, Universidad Nacional del Nordeste, Universidad Metropolitana para la Educación y el Trabajo y Universidad Nacional de Moreno. Recuperado de http://biblioteca.clacso.edu.ar/Argentina/foumet/20160212070619/Neffa.pdf

Neffa, J.C. (2017). Ley de Riesgos del Trabajo. Los nuevos desafíos. En Neffa, J.C., Una reforma que no resuelve los problemas de los riesgos del trabajo en Argentina: debate interdisciplinario en la UMET. Universidad Metropolitana para la Educación y el Trabajo (UMET). Recuperado de https://www.researchgate.net/publication/32111 0480_Una_reforma_que_no_resuelve_los_prob lemas_de_los_riesgos_del_trabajo_en_Argentin a_debate_interdisciplinario_en_la_UMET/link/ 5a0dee3645851541b7079e62/download

Organización Internacional del Trabajo. (2013a). La organización del trabajo y los riesgos psicosociales: una mirada de género. Recuperado de https://www.ilo.org/wcmsp5/groups/public/--americas/---ro-lima/---srosan_jose/documents/publication/wcms_227402. pdf 
Organización Internacional del Trabajo. (2013b). La prevención de las enfermedades profesionales. Recuperado de https://www.ilo.org/wcmsp5/groups/public/--ed_protect/---protrav/---

safework/documents/publication/wcms_209555 .pdf

Palomo, G., Carrasco, J., Bastías, A., Méndez, M. D. y Jiménez, A. (2015). Factores de riesgo psicosocial y satisfacción laboral en trabajadoras estacionales de Chile. Rev Panam Salud Pública, 37 (4/5), 301-307. Recuperado de https://www.scielosp.org/pdf/rpsp/2015.v37n45/301-307

Posada, J. I., Molano, P. N., Parra, R. M., Brito, F. Y. y Rubio, E. A. (2019). Prevalencia del Síndrome de Burnout en docentes: Factores asociados al estatuto de vinculación laboral en Colombia. Revista Interamericana de Psicología Ocupacional, 37 (2), 119-133. Recuperado de https://www.proquest.com/docview/230619485 4/B9D249D6D9014135PQ/1

Potter, R., O’Keeffe, V., Leka, S., Webber, M., y Dollard, M. (2019). Analytical review of the australian policy context for work-related psychological health and psychosocial risks. Safety Science, 111, 37-48. Recuperado de https://www.sciencedirect.com/science/article/p ii/S092575351830242X?via\%3Dihub

Rodríguez, M. (2009). Factores Psicosociales de Riesgo Laboral: ¿Nuevos tiempos, nuevos riesgos? Observatorio Laboral Revista Venezolana, 2 (3), 127-141. Recuperado de https://dialnet.unirioja.es/servlet/articulo?codig o=2995368

Ruiz, P., Pullas, P., Parra, C. A. y Zamora, R. (2017). La doble presencia en las trabajadoras femeninas: equilibrio entre el trabajo y la vida familiar. Revista de Comunicación de la SEECI, (44), 33-51. Recuperado de https://www.proquest.com/docview/201352905 5/A2F654A56E264CFDPQ/1

Salamanca, S. R., Pérez, J. M., Infante, A. F. y Olarte, Y. Y. (2019). Análisis de los factores de riesgo psicosocial a nivel nacional e internacional. Revista TEMAS, 3 (13), 39-45. Recuperado de https://dialnet.unirioja.es/servlet/articulo?codig $\mathrm{o}=7169071$
Saldaña, C; Polo, J. D.; Gutiérrez, O. I. y Madrigal, B. E. (2020). Bienestar psicológico, estrés y factores psicosociales en trabajadores de instituciones gubernamentales de Jalisco, México. Revista de Ciencias Sociales, 26 (1), 25-37. Recuperado de http://www.redalyc.org/articulo.oa? $\mathrm{id}=2806310$ 4003

Secretaría del Trabajo y Previsión Social (STPS). (2019). Norma Oficial Mexicana NOM035-STPS-2018. Factores de riesgo psicosocial en el trabajo - Identificación, análisis y prevención. Recuperado de https://www.gob.mx/stps/articulos/normaoficial-mexicana-nom-035-stps-2018-factoresde-riesgo-psicosocial-en-el-trabajoidentificacion-analisis-y-prevencion

Seijas-Solano, D. (2019). Riesgos psicosociales, estrés laboral y síndrome burnout en trabajadores universitarios de una escuela de bioanálisis. Revista Salud Pública, 21 (1), 102108. Recuperado de https://www.proquest.com/docview/232959010 2/691BAE45CA5747DDPQ/1

Tacca, D. R. y Tacca, A. L. (2019). Factores de riesgos psicosociales y estrés percibido en docentes universitarios. Propósitos y Recomendaciones, 7 (3), 323-353. Recuperado de

http://www.scielo.org.pe/pdf/pyr/v7n3/a13v7n3 .pdf

Toia, M., Mattos, M., Figueroa, G., Aguilar, E., Chavez, V., Del Carpio, B., Gamarra, S., García, K., Miranda, G., Romero, L. y Muñoz, A. (2018). Factores psicosociales en el trabajo y su relación con la doble presencia de las trabajadoras mujeres en servicios de atención primaria de la salud. Revista Postgrado Scientiarvm,4 (2), 13-18. Recuperado de http://scientiarvm.org/cache/archivos/PDF_674 125980.pdf

Uribe-Prado, J. F. (2020). Riesgos psicosociales, burnout y psicosomáticos en trabajadores del sector público. Investigación Administrativa, 49 (125). Recuperado de https://www.redalyc.org/articulo.oa?\%2520id= 456061607013 
Villamar, D., Juárez, A., González, I. G. y Osnaya, M. (2019). Factores psicosociales y síndrome de Burnout en académicos de una universidad pública de México. Propósitos y Recomendaciones, 7 (3), 11-140. Recuperado de http://www.scielo.org.pe/pdf/pyr/v7n3/a05v7n3 .pdf 